\title{
ON THE NUMBER AND SUM OF NEAR-RECORD OBSERVATIONS
}

\author{
N. BALAKRISHNAN, ${ }^{*}$ McMaster University
}

A. G. PAKES, ${ }^{* *}$ The University of Western Australia

A. STEPANOV, ${ }^{* * *}$ Kaliningrad State Technical University

\begin{abstract}
Let $X_{1}, X_{2}, \ldots$ be a sequence of independent and identically distributed random variables with some continuous distribution function $F$. Let $L(n)$ and $X(n)$ denote the $n$th record time and the $n$th record value, respectively. We refer to the variables $X_{i}$ as near-nthrecord observations if $X_{i} \in(X(n)-a, X(n)]$, with $a>0$, and $L(n)<i<L(n+1)$. In this work we study asymptotic properties of the number of near-record observations. We also discuss sums of near-record observations.
\end{abstract}

Keywords: Record; near-record observation; record time; sum of near-record observations; insurance claim; limit theorem

2000 Mathematics Subject Classification: Primary 60G70; 62G30

\section{Introduction}

Let $X_{1}, X_{2}, \ldots$ be independent and identically distributed (i.i.d.) random variables (RVs). The sequences of record values $X(n)$ and record times $L(n)$ are defined as follows:

$$
\begin{aligned}
L(1) & =1, \\
L(n+1) & =\min \left\{j: j>L(n), X_{j}>X_{L(n)}\right\}, \\
X(n) & =X_{L(n)} \quad(n \geq 1) .
\end{aligned}
$$

If in (1.1) the second condition on $j$ is replaced by $X_{j} \geq X_{L(n)}$, then the above definitions become the definitions of weak record values $X^{\mathrm{w}}(n)$ and weak record times $L^{\mathrm{w}}(n)$. Note that there is no difference between records and weak records when the initial distribution function $F(x)=\mathrm{P}\left\{X_{1} \leq x\right\}$ is continuous; see Arnold et al. (1998) and Nevzorov (2000).

The distribution function of $X^{\mathrm{w}}(n)$ in the discrete case was derived by Stepanov (1992) using the auxiliary variables $\xi_{i}$, defined as follows. Suppose that $F$ is concentrated on nonnegative integers and $F(m)<1$ for any $m \geq 0$. Let the variable $\xi_{i}, i \geq 0$, count the number of weak record values that belong to the point $i$, i.e. $\xi_{i}=k, k \geq 0$, if exactly $k$ weak record values have been registered at $i$. The following lemma was given in Stepanov (1992).

\footnotetext{
Received 29 March 2004; revision received 9 November 2004.

* Postal address: Department of Mathematics and Statistics, McMaster University, 1280 Main Street West, Hamilton, Ontario L8S 4K1, Canada. Email address: bala@univmail.cis.mcmaster.ca

** Postal address: Department of Mathematics, The University of Western Australia, 35 Stirling Highway, Crawley, WA 6009, Australia. Email address: pakes@maths.uwa.edu.au

*** Postal address: Department of Mathematics, Kaliningrad State Technical University, Sovietsky Prospect 1, Kaliningrad, 236000, Russia. Email address: alexei@step.koenig.ru
} 
Lemma 1.1. The variables $\xi_{0}, \xi_{1}, \ldots$ are independent and $\mathrm{P}\left\{\xi_{i}=k\right\}=p_{i} q_{i}^{k}, k \geq 0$, where

$$
1-p_{i}=q_{i}=\frac{\mathrm{P}\left\{X_{1}=i\right\}}{\mathrm{P}\left\{X_{1} \geq i\right\}} .
$$

In addition,

$$
\mathrm{P}\left\{X^{\mathrm{W}}(n)>m\right\}=\mathrm{P}\left\{\xi_{0}+\cdots+\xi_{m}<n\right\}
$$

Recently, Stepanov et al. (2003) derived the distribution function of $X^{\mathrm{w}}(n)$ for the case in which $F$ is arbitrary.

The works of Eisenberg et al. (1993), Brands et al. (1994), Baryshnikov et al. (1995), Qi (1997), and others have concentrated on the problem of 'a tie for first place'. Let $X_{1}, X_{2}, \ldots$ be i.i.d. integer-valued RVs, let $M_{n}=\max \left\{X_{1}, X_{2}, \ldots, X_{n}\right\}$, and let $K_{n}=\sum_{i=1}^{n} \mathbf{1}\left(X_{i}=M_{n}\right)$. All the authors just mentioned have studied the behaviour of $K_{n}$, which is the number of sample observations achieving the sample maximum in the discrete case. As a natural continuous analogue of $K_{n}$, Pakes and Steutel (1997) considered the variable

$$
K_{n}(a)=\operatorname{card}\left\{j: X_{j} \in\left(M_{n}-a, M_{n}\right]\right\}
$$

where $a>0$ and $X_{1}, X_{2}, \ldots, X_{n}$ are i.i.d. from some continuous distribution. The variable $K_{n}(a)$ is then the number of near-maximum observations, i.e. the number of observations in the sample that have been registered in the random interval $\left(M_{n}-a, M_{n}\right]$. Since Pakes and Steutel (1997), the behaviour of $K_{n}(a)$ has been studied in detail by many authors, including Li (1999), Pakes (2000), Hu and Su (2003), and Balakrishnan and Stepanov (2005).

Acknowledging the similarity in approaches to studying maximum values and record values, and following the lead of Pakes and Steutel (1997), who suggested $K_{n}(a)$ as a continuous analogue of $K_{n}$, here we study counting variables that comprise a continuous analogue of the variables $\xi_{n}$ defined above.

Let $X_{1}, X_{2}, \ldots$ be i.i.d. RVs with a continuous distribution function $F(x)$. Fix $a>0$ and say that $X_{j}$ is a near- $n$ th-record if $L(n)<j<L(n+1)$ and $X_{j} \in(X(n)-a, X(n)]$. We define the number of near- $n$ th-records to be

$$
\xi_{n}(a)=\operatorname{card}\left\{j: L(n)<j<L(n+1), X_{j} \in(X(n)-a, X(n)]\right\} \quad(n \geq 1) .
$$

Observe that

$$
0 \leq \xi_{n}(a) \leq L(n+1)-L(n)-1=: \Delta(n+1)-1,
$$

and that $\xi_{n}(0)=0$ and $\xi_{n}(\infty)=\Delta(n+1)-1$. The variable $\Delta(n)$ is referred to in the theory of records as the interrecord time.

In this paper we study properties of the $\xi_{n}(a)$ in the case of continuous $F$. Basic distributional results for $\xi_{n}(a)$ are given in Section 2. In Section 3, the number of near-record observations is investigated in the case in which the right-extremity of $F, r_{F}=\inf \{x: F(x)=1\}$, is finite. Asymptotic results for $\xi_{n}(a)$ in the case $r_{F}=\infty$ are presented in Section 4 , and results for sums of near-record observations, in the same case, are presented in Section 5. We also mention possible insurance applications of the results in Section 5. Finally, some examples are presented in Section 6. 


\section{Distributional results for the number of near-record observations}

In the sequel, we denote the survivor function $1-F(x)$ by $\bar{F}(x)$.

Theorem 2.1. The probability mass function of $\xi_{n}(a), a>0, n \geq 1$, is given by

$$
\mathrm{P}\left\{\xi_{n}(a)=k\right\}=\int_{\mathbb{R}} \beta(x, a)\{1-\beta(x, a)\}^{k} \mathrm{~d} F_{n}(x) \quad(k \geq 0),
$$

where

$$
F_{n}(x)=\frac{1}{(n-1) !} \int_{0}^{-\log \bar{F}(x)} \mathrm{e}^{-u} u^{n-1} \mathrm{~d} u
$$

is the distribution function of $X(n)$ and

$$
0 \leq \beta(x, a)=\frac{\bar{F}(x)}{\bar{F}(x-a)} \leq 1 .
$$

Proof. First, we have

$$
\mathrm{P}\left\{\xi_{n}(a)=k\right\}=\int_{\mathbb{R}} \mathrm{P}\left\{\xi_{n}(a)=k \mid X(n)=x\right\} \mathrm{d} F_{n}(x) .
$$

In order to estimate the conditional probability $\mathrm{P}\left\{\xi_{n}(a)=k \mid X(n)=x\right\}$, we employ Nevzorov's (1986) deletion argument. From the sequence of independent $X_{i}, i>L(n)$, we delete all those $X_{i}$ for which $X_{i} \leq x-a$, leaving the sequence $X_{i_{1}}, X_{i_{2}}, \ldots, L(n)<i_{1}<i_{2}<\cdots$, comprising only those $X_{i}$ that are equal to at least $x-a$. This deletion procedure does not alter the number of near-records in the interval $(x-a, x]$. Let $Y_{j}, j \geq 1$, denote random variables that are conditionally independent given $X(n)=x$ and such that

$$
\mathrm{P}\left\{Y_{j} \leq y\right\}=\mathrm{P}\left\{X_{i_{j}} \leq y \mid X_{i_{j}} \geq x-a\right\} \text { for } y \geq x-a .
$$

Then

$$
\mathrm{P}\left\{\xi_{n}(a)=k \mid X(n)=x\right\}=\mathrm{P}\left\{Y_{1} \leq x, \ldots, Y_{k-1} \leq x, Y_{k}>x\right\},
$$

which readily yields $(2.1)$.

Theorem 2.1 asserts that, conditionally on $X(n)=x$, the number of near-records has a geometric distribution with parameter $\beta(x, a)$. This is dual to the result, used by Pakes and $\mathrm{Li}$ (1998), stating that, conditionally on the sample maximum, the number of near-maxima has a shifted binomial distribution.

Theorem 2.2. The probability mass functions of

$$
\left(\xi_{n}\left(a_{1}\right), \ldots, \xi_{n+t-1}\left(a_{t}\right)\right) \text { and }\left(\xi_{n}\left(a_{1}\right), \xi_{n+t-1}\left(a_{t}\right)\right),
$$

for $a_{1}>0, \ldots, a_{t}>0, k_{1} \geq 0, \ldots, k_{t} \geq 0$, and $t \geq 2$ are respectively

$$
\begin{aligned}
& \mathrm{P}\left\{\xi_{n}\left(a_{1}\right)=k_{1}, \ldots, \xi_{n+t-1}\left(a_{t}\right)=k_{t}\right\} \\
& =\int_{\mathbb{R}} \int_{x_{n}}^{\infty} \cdots \int_{x_{n+t-2}}^{\infty} \beta\left(x_{n}, a_{1}\right)\left\{1-\beta\left(x_{n}, a_{1}\right)\right\}^{k_{1}} \cdots \beta\left(x_{n+t-1}, a_{t}\right) \\
& \quad \times\left\{1-\beta\left(x_{n+t-1}, a_{t}\right)\right\}^{k_{t}} \mathrm{~d} H_{1}\left(x_{n+t-1}, x_{n+t-2}\right) \cdots \mathrm{d} H_{1}\left(x_{n+1}, x_{n}\right) \mathrm{d} F_{n}\left(x_{n}\right)
\end{aligned}
$$


and

$$
\begin{aligned}
& \mathrm{P}\left\{\xi_{n}\left(a_{1}\right)=k_{1}, \xi_{n+t-1}\left(a_{t}\right)=k_{t}\right\} \\
& =\int_{\mathbb{R}} \int_{x_{n}}^{\infty} \beta\left(x_{n}, a_{1}\right)\left\{1-\beta\left(x_{n}, a_{1}\right)\right\}^{k_{1}} \beta\left(x_{n+t-1}, a_{t}\right)\left\{1-\beta\left(x_{n+t-1}, a_{t}\right)\right\}^{k_{t}} \\
& \quad \times \mathrm{d} H_{t-1}\left(x_{n+t-1}, x_{n}\right) \mathrm{d} F_{n}\left(x_{n}\right),
\end{aligned}
$$

where

$$
H_{t}(y, x)=\frac{1}{(t-1) !} \int_{0}^{-\log (\bar{F}(y) / \bar{F}(x))} \mathrm{e}^{-u} u^{t-1} \mathrm{~d} u \quad(y \geq x) .
$$

Proof. These results can be proved by means of the deletion argument used to prove Theorem 2.1.

\section{The case of finite right-extremity}

Although it is obvious that the number of record values grows unboundedly, it is less obvious that the number of near-record values occurring between successive record times grows unboundedly. The following result shows that this is in fact the case if $r_{F}<\infty$, and gives some idea of the growth rate. We denote a nonnegative random variable having the standard exponential density function $\mathrm{e}^{-x}$ by $\mathcal{E}$.

Theorem 3.1. Suppose that $F$ is continuous and $r_{F}<\infty$. Then,

(i) for every fixed $a>0$,

$$
\left.\xi_{n}(a) \rightarrow \infty \quad(\text { almost surely (a.s. })\right) \quad(n \rightarrow \infty)
$$

(ii) $\bar{F}(X(n)) \xi_{n}(a) \stackrel{\mathrm{D}}{\rightarrow} \omega \mathcal{E}$, where $\omega=\bar{F}\left(r_{F}-a\right)$; and

(iii) $n^{-1} \log \xi_{n}(a) \stackrel{\mathrm{P}}{\rightarrow} 1$.

Here, ' $\stackrel{\mathrm{D}}{\rightarrow}$ ' and $\stackrel{\mathrm{P}}{\rightarrow}$ ' denote convergence in distribution and in probability, respectively.

Proof. Formula (2.1) yields

$$
\mathrm{P}\left\{\xi_{n}(a) \leq k\right\}=\int_{\mathbb{R}}\left[1-\{1-\beta(x, a)\}^{k+1}\right] \mathrm{d} F_{n}(x) .
$$

Expressing the integrand in terms of $A=\bar{F}(x)$ and $B=\bar{F}(x-a)$, and using the bound $B^{k+1}-(B-A)^{k+1} \leq(k+1) A B^{k}$, yields the crude bound

$$
\mathrm{P}\left\{\xi_{n}(a) \leq k\right\} \leq(k+1) \int_{\mathbb{R}} \frac{\bar{F}(x)}{\bar{F}(x-a)} \mathrm{d} F_{n}(x) .
$$

Since $\sum_{n \geq 1} \mathrm{~d} F_{n}(x)=\mathrm{d} F(x) / \bar{F}(x)$, we obtain the inequality

$$
\sum_{n=1}^{\infty} \mathrm{P}\left\{\xi_{n}(a) \leq k\right\} \leq(k+1) \int_{\mathbb{R}} \frac{\mathrm{d} F(x)}{\bar{F}(x-a)} .
$$

The right-hand integral is bounded above by $1 / \bar{F}\left(r_{F}-a\right)<\infty$, and (3.1) follows from the first Borel-Cantelli lemma. 
Theorem 2.1 implies that the probability generating function of $\xi_{n}(a)$ is

$$
\mathrm{E}\left[s^{\xi_{n}(a)}\right]=\mathrm{E}\left[\frac{\beta(X(n), a)}{1-s(1-\beta(X(n), a))}\right] .
$$

However, $\beta(X(n), a) \sim \bar{F}(X(n)) / \omega$ almost surely as $n \rightarrow \infty$, so, upon setting

$$
s=\mathrm{e}^{-\theta \bar{F}(X(n))}=1-\theta \bar{F}(X(n))(1+o(1)) \quad \text { a.s., for } \theta>0,
$$

we see that the term in square brackets in (3.2) converges almost surely to $(1+\omega \theta)^{-1}$, the Laplace-Stieltjes transform of $\omega \mathcal{E}$. Thus, part (ii) follows from the dominated convergence theorem.

The known representation (Nevzorov (2000, pp. 66, 69))

$$
-\log \bar{F}(X(n)) \stackrel{\mathrm{D}}{=} \zeta_{1}+\cdots+\zeta_{n},
$$

where the summands are independent and have the standard exponential distribution, together with the weak law of large numbers imply that $(-\log \bar{F}(X(n))) / n \stackrel{\mathrm{P}}{\rightarrow} 1$, and part (iii) follows from part (i).

Remark 3.1. Extending the proof of part (ii) using the central limit theorem implies that

$$
\frac{\log \xi_{n}(a)-n}{\sqrt{n}} \stackrel{\mathrm{D}}{\rightarrow} N(0,1),
$$

but part (iii) provides the best description of the asymptotic growth of $\xi_{n}(a)$.

Let us compare the case in which $F$ is continuous and $r_{F}<\infty$ with the discrete analogue of this situation, i.e. in which $X_{1}, X_{2}, \ldots$ are i.i.d. integer-valued variables concentrated on $0,1, \ldots, m$. In this case, it is known that $\xi_{m}=\infty$ a.s.; see Arnold et al. (1998) or Stepanov (1992).

It is also interesting to look at the asymptotic behaviour of $\Delta(n)$ in the context of inequality (1.2) and Theorem 3.1. The theory of records states that $\Delta(n) \sim \mathrm{e}^{n}$ a.s. $($ as $n \rightarrow \infty)$ for any continuous distribution function $F$; see, for example, Galambos (1987).

\section{Limiting results with infinite extremity}

For the remainder of this paper, we take $r_{F}=\infty$ and we assume the existence of the limit

$$
\lim _{x \rightarrow \infty} \beta(x, a)=\lim _{x \rightarrow \infty} \beta(x+a, a)=\beta(a) .
$$

Clearly $0 \leq \beta(a) \leq 1$. Assuming that this limit exists for all positive $a$, Pakes and Steutel (1997) used it to classify distribution tails as 'thin' if $\beta(a)=0$, 'medium' if $0<\beta(a)<1$, and 'thick' if $\beta(a)=1$.

Remark 4.1. At first sight, it appears that the existence of (4.1) for one value of $a>0$ entails its existence for all $a>0$. Balakrishnan and Stepanov (2005) gave an example showing that this is not the case. A result of Karamata (Bingham et al. (1987, p. 54)) implies that (4.1) holds for all $a>0$ if and only if it holds for an arbitrary pair $a_{1}, a_{2}>0$ such that $a_{1} / a_{2}$ is irrational.

In the sequel, $G(p)$ denotes a random variable having the geometric distribution with mass $p(1-p)^{k}$ at $k=0,1, \ldots$ 
Theorem 4.1. Assume that $F$ is continuous, that $r_{F}=\infty$, and that (4.1) holds for some a $>0$. Then, as $n \rightarrow \infty$,

(i) $\xi_{n}(a) \stackrel{\mathrm{P}}{\rightarrow} \infty$ if $\beta(a)=0$,

(ii) $\xi_{n}(a) \stackrel{\mathrm{D}}{\rightarrow} G(\beta(a))$ if $0<\beta(a)<1$, and

(iii) $\xi_{n}(a) \stackrel{\mathrm{P}}{\rightarrow} 0$ if $\beta(a)=1$.

Proof. We first prove part (ii). If $F(x)>0$ then

$$
F_{n}(x) \leq-\frac{\log \bar{F}(x)}{n !} \rightarrow 0 \quad(n \rightarrow \infty) .
$$

It follows from (4.1) that if $k \geq 0$ and $x_{0}$ is sufficiently large, (2.1) can be expressed as

$$
\mathrm{P}\left\{\xi_{n}(a)=k\right\}=\int_{-\infty}^{x_{0}} \beta(x, a)\{1-\beta(x, a)\}^{k} \mathrm{~d} F_{n}(x)+\int_{x_{0}}^{\infty} \beta(a)\{1-\beta(a)\}^{k} \mathrm{~d} F_{n}(x)+o(1) .
$$

The first integral on the right-hand side is bounded above by $F_{n}\left(x_{0}\right)$, whence part (ii) follows from (4.2). Trivial alterations give parts (i) and (iii).

By applying (4.2) for $\mathrm{E}\left[\xi_{n}(a)\right]=\int_{\mathbb{R}}\{(1-\beta(x, a)) / \beta(x, a)\} \mathrm{d} F_{n}(x)$, we obtain the following result.

Theorem 4.2. Assume that $F$ is continuous, that $r_{F}=\infty$, and that (4.1) holds for some $a>0$.

(i) If $\beta(a)=0$ then $E \xi_{n}(a) \rightarrow \infty$.

(ii) If $0<\beta(a)<1$ then $E \xi_{n}(a) \rightarrow(1-\beta(a)) / \beta(a)$.

(iii) If $\beta(a)=1$ then $E \xi_{n}(a) \rightarrow 0$.

These statements can be explained in the following manner. For a thin-tailed distribution $(\beta(a) \equiv 0)$, the record value $X(n)$ jumps at the 'beginning' of the tail of the distribution, i.e. the value of $X(n)$ is comparatively small. Consequently, the random interval $(X(n)-a, X(n)]$ contains an almost constant portion of the growing number of observations $X_{L(n)+1}, \ldots, X_{L(n+1)-1}$, whose number, $\Delta(n)-1$, tends to $\infty$ as $n \rightarrow \infty$. Consequently, $\xi_{n}(a)$ also tends to $\infty$. On the other hand, for a thick-tailed distribution the gaps $X(n)-X(n-1)$ become increasingly large. Consequently, $(X(n)-a, X(n)]$ tends to be empty with high probability.

Our next principal results are strong law versions of parts (i) and (iii) of Theorem 4.1.

Theorem 4.3. Assume that $F$ is continuous, that $r_{F}=\infty$, and, for some fixed $b>0$, that

$$
I(b)=\int_{\mathbb{R}} \frac{\bar{F}(x-b)-\bar{F}(x)}{\bar{F}^{2}(x)} \mathrm{d} F(x)<\infty .
$$

Then

(a) $\beta(a)=1$ and $\xi_{n}(a) \stackrel{\mathrm{P}}{\rightarrow} 0$ for any $a>0$, and

(b) $\xi_{n}(a) \rightarrow 0$ a.s. if $0<a \leq b$. 
Proof. (a) Li (1999) has shown that (4.3) implies that the number of near-maxima $K_{n}(b) \rightarrow 1$ a.s. It follows that $\beta(b)=1$, since Pakes and Steutel (1997) showed this to be necessary and sufficient for convergence in probability. Hence, $\beta(a)=1$ if $a \in[0, b]$ and, if $b<a \leq 2 b$, then $\beta(x, a)=\beta(x, b) \beta(x-b, a-b) \rightarrow 1$. The first assertion of part (a) follows by induction, and the second from Theorem 4.1(iii).

(b) Next, it is clear that $I(a)<\infty$ if $a \leq b$, and it follows from (2.1) that

$$
\sum_{n=1}^{\infty} \mathrm{P}\left\{\xi_{n}(a)>0\right\}=\int_{\mathbb{R}} \frac{\bar{F}(x-a)-\bar{F}(x)}{\bar{F}(x) \bar{F}(x-a)} \mathrm{d} F(x) .
$$

Since $\beta(a)=1$, it follows that this integral converges if and only if (4.3) holds. Assertion (b) follows from the first Borel-Cantelli lemma.

Suppose that (4.3) holds. The question of whether $I(a)<\infty$ for all $b>a$ appears to be related to O-versions of de Haan theory (Bingham et al. (1987, Chapter 3)). To explore this, we introduce some notation for regular variation. Denote by $\mathcal{R}_{d}$ the set of (measurable) functions $R(x)$ that are regularly varying (at infinity) with index $d$ (a real number), i.e. for all $\lambda>0$, we have $\lim _{x \rightarrow \infty} R(\lambda x) / R(x)=\lambda^{d}$. This implies the representation $R(x)=x^{d} \mathcal{L}(x)$ (for $x>0$ ), where $\mathcal{L}(x)$ is slowly varying, i.e. $\mathcal{L}(x) \in \mathcal{R}_{0}$. A slowly varying function has the canonical form

$$
\mathcal{L}(x)=C(x) \exp \left[\int_{1}^{x} \frac{\epsilon(y)}{y} \mathrm{~d} y\right],
$$

where $C(x) \rightarrow C>0$ and $\epsilon(x)$ is an index function such that $\epsilon(x) \rightarrow 0$ as $x \rightarrow \infty$. We say that $\mathcal{L}(x)$ is normalized slowly varying if $C(x) \equiv C$, and we denote this subclass by $\overline{\mathcal{R}}_{0}$. Finally, we say that $R(x)$ is extended regularly varying, $R(x) \in E \mathcal{R}$, if there exist real constants $c$ and $d, c \geq d$, such that

$$
\lambda^{-c} \leq \liminf _{x \rightarrow \infty} R(\lambda x) / R(x) \leq \limsup _{x \rightarrow \infty} R(\lambda x) / R(x) \leq \lambda^{-d} \quad(0<\lambda \leq 1) .
$$

This is an adaptation of the definition given by Bingham et al. (1987, p. 65) and is better suited to our needs.

With reference to Theorem 4.3(a), observe that $\beta(a) \equiv 1$ if and only if $\bar{F}(x)=1 / \mathcal{L}\left(\mathrm{e}^{x}\right)$, where $\mathcal{L}(y) \in \mathcal{R}_{0}$. Observe that $\int_{1}^{\infty}(\epsilon(y) / y) \mathrm{d} y=\infty$ since $\bar{F}(\infty)=0$; thus, if $\epsilon(x) \in \mathcal{R}_{-d}$ then $0 \leq d \leq 1$. We can now state the following addendum to Theorem 4.3.

Lemma 4.1. Suppose that $\bar{F}(x)=1 / \mathcal{L}\left(\mathrm{e}^{x}\right)$, where $\mathcal{L} \in \overline{\mathcal{R}}_{0}$ has index function $\epsilon(x) \in E \mathcal{R}$. Then either $I(a)<\infty$ for all $a>0$ or for no a $>0$. In the first case, $\xi_{n}(a) \rightarrow 0$ (a.s.) for all $a>0$.

Proof. The condition $\mathcal{L}(y) \in \overline{\mathcal{R}}_{0}$ implies that, for any $a>0$,

$$
\frac{\bar{F}(x-a)}{\bar{F}(x)}-1 \sim \int_{\exp [x-a]}^{\exp [x]} \frac{\epsilon(y)}{y} \mathrm{~d} y .
$$

The uniform convergence theorem for extended regular variation (Bingham et al. (1987, p. 66), altered for $\lambda \leq 1)$ can be used to show that, for all sufficiently large $x$, the integral is bounded between $a \epsilon\left(\mathrm{e}^{x}\right)(1+o(1))$ and $a \epsilon\left(\mathrm{e}^{x}\right) \mathrm{e}^{a d}(1+o(1))$. It follows that

$$
\frac{a}{b} \mathrm{e}^{-b c} \leq \liminf _{x \rightarrow \infty} \frac{\bar{F}(x-a)-\bar{F}(x)}{\bar{F}(x-b)-\bar{F}(x)} \leq \limsup _{x \rightarrow \infty} \frac{\bar{F}(x-a)-\bar{F}(x)}{\bar{F}(x-b)-\bar{F}(x)} \leq \frac{a}{b} \mathrm{e}^{a d}
$$


and, hence, $I(a)<\infty$ if and only if $I(b)<\infty$. This proves the first assertion, and the second follows from Theorem 4.3.

We conclude that if (4.3) holds for all $b>0$ then, for any $a>0$, there is a random variable $N_{a}$ such that $\xi_{n}(a) \equiv 0$ if $n \geq N_{a}$, i.e. all observations occurring between $L(n)$ and $L(n+1)$ are smaller than $X(n)-a$, and, hence, that attaining the record value $X(n+1)$ implies that $X(n+1)-X_{j}>a$ if $L(n)<j<L(n+1)$ and $n \geq N_{a}$.

Pakes (2005) gave conditions ensuring that (4.3) holds for all $b>0$. One is that $\bar{F}(x) \in \mathcal{R}_{-\alpha}$ for $\alpha \geq 0$. Also, if $\bar{F}(x)=\exp [-R(x)]$, where $R(x) \in \mathcal{R}_{\alpha}$, then (4.3) holds if $0 \leq \alpha<\frac{1}{2}$, and does not hold if $\alpha>\frac{1}{2}$.

Our next result gives a condition allowing us to assert almost-sure convergence in Theorem 4.1(i).

Theorem 4.4. Assume that $F$ is continuous, that $r_{F}=\infty$, and, for some fixed $b>0$, that

$$
J(b)=\int_{\mathbb{R}} \frac{\mathrm{d} F(x)}{\bar{F}(x-b)}<\infty .
$$

Then

(a) $\beta(a)=0$ for all $a>0$, and

(b) if $a \geq b$ then $\xi_{n}(a) \rightarrow \infty$ a.s.

Proof. (a) The integrand in (4.4) is nondecreasing and, hence, $\bar{F}(x) / \bar{F}(x-b) \rightarrow 0$, i.e. $\beta(a)=0$ if $a \geq b$. If $a<b$ then

$$
\beta(x, a)=\beta(x-b, a+b) \frac{\bar{F}(x)}{\bar{F}(x-b)} \leq \beta(x-b, a+b) \rightarrow 0 .
$$

(b) Observe that (2.1) yields

$$
\sum_{n=1}^{\infty} \mathrm{P}\left\{\xi_{n}(b) \leq k\right\}=\int_{\mathbb{R}}\left[1-(1-\beta(x, b))^{k}\right] \frac{\mathrm{d} F(x)}{\bar{F}(x)},
$$

and the assertion follows from (4.4) because the integrand $\sim k / \bar{F}(x-b)$.

The condition (4.4) should not be difficult to check in specific cases. However, the necessary condition (a) in the theorem is equivalent to having the representation $\bar{F}(x)=1 / r\left(\mathrm{e}^{x}\right)$, where $r(y)$ is rapidly varying, i.e. $\lim _{y \rightarrow \infty} r(\lambda y) / r(y)=\infty$ if $\lambda>1$ or $\lim _{y \rightarrow \infty} r(\lambda y) / r(y)=0$ if $0<\lambda<1$; see Bingham et al. (1987, p. 83). We denote this by writing $r(y) \in \mathcal{R}_{\infty}$. A representation theorem asserts that $r(y)=C(y) \exp \left[\int_{1}^{y}(\delta(z) / z) \mathrm{d} z\right]$, where $\delta(y) \rightarrow \infty$ and $C(y) \rightarrow C>0$ as $y \rightarrow \infty$. The subclass $\overline{\mathcal{R}}_{\infty}$ of normalized rapidly varying functions satisfies $C(y) \equiv C$. For later reference, we collectively denote reciprocals of these functions by $\mathcal{R}_{-\infty}$ and $\overline{\mathcal{R}}_{-\infty}$, respectively. The following theorem gives some sufficient conditions for (4.4) to hold.

Theorem 4.5. (a) Suppose that $\bar{F}(x)=1 / r\left(\mathrm{e}^{x}\right)$, where $r(y) \in \overline{\mathcal{R}}_{\infty}$ with index function $\delta(y) \in E \mathcal{R}$ such that $\int_{1}^{\infty}\left[y(\delta(y))^{v}\right]^{-1} \mathrm{~d} y<\infty$ for some $v>0$. Then (4.4) holds for all $b>0$. 
(b) In particular, these conditions are satisfied if $\bar{F}(x)=\exp [-R(x)]$, where $R(x) \in \overline{\mathcal{R}}_{\ell}$ and $v \ell>1$.

Proof. The integral representation of $r(y)$ implies that $\mathrm{d} F(x) / \mathrm{d} x=\delta\left(\mathrm{e}^{x}\right) / r\left(\mathrm{e}^{x}\right)$ and, hence, $J(b)<\infty$ if

$$
\hat{J}(b):=\int_{1}^{\infty} \frac{r\left(y \mathrm{e}^{-b}\right)}{y r(y)} \delta(y) \mathrm{d} y<\infty .
$$

Our definition of extended regular variation implies that if $0<\epsilon<1$ then $y^{\prime}$ can be chosen so large that

$$
r\left(y \mathrm{e}^{-b}\right) / r(y) \leq \exp [-\eta \delta(y)] \quad\left(y \geq y^{\prime}\right),
$$

where $\eta=(1-\epsilon) b \mathrm{e}^{b c}$. Assertion (a) follows because, for any $v>0$, the last bound is dominated by $(\eta \delta(y))^{-v-1}$. To prove part (b), observe that $r(y)=\exp [R(\log y)]$ and that $R(\lambda z)-R(z) \rightarrow \infty$ (as $z \rightarrow \infty)$ if $\lambda>1$, whence $r(y) \in \overline{\mathcal{R}}_{\infty}$. The index function $\delta(y)$ of $r(y)$ satisfies $\delta(y) / y=(\mathrm{d} / \mathrm{d} y) R(\log y)$, from which we find that $\delta(y) \sim \ell R(\log y) / \log y \in \mathcal{R}_{0}$. Also, $\delta(y) \geq \ell(\log y)^{\gamma}$, where $0<\gamma<\ell-1$, and $\int_{1}^{\infty} y^{-1}(\log y)^{-v \gamma} \mathrm{d} y<\infty$ if $\nu \gamma>1$.

In Example 6.6, we will show that a thin-tailed distribution function need not satisfy (4.4), or that it may do for some values of $b$ and not for others.

\section{Sums of near-record observations}

We assume, in this section, that $X_{i}, i \geq 1$, take on only positive values and that $r_{F}=\infty$. Let $s_{n}(a)$ be the sum of near- $n$ th-record observations, i.e.

$$
s_{n}(a)=\sum_{i=L(n)+1}^{L(n+1)-1} X_{i} \mathbf{1}\left(X_{i} \in(X(n)-a, X(n)]\right) .
$$

This sum is a quantity of interest because it can be interpreted as the sum of insurance claims close in value to, and following the occurrence of, an unusually large claim, $X(n)$. The registration of these claims is stopped at the next record time $L(n+1)$. Pakes (2000), Li and Pakes (2001), and Hashorva (2003) discussed similar aspects for near-maximum observations. Arnold and Villasenor (1998) have studied sums of record values.

The limiting behaviour of $s_{n}(a)$ can easily be found from the results presented in the last section and the inequality

$$
(X(n)-a) \xi_{n}(a)<s_{n}(a) \leq X(n) \xi_{n}(a),
$$

which holds on almost all sample paths. Recalling that $r_{F}=\infty$, we have $X(n) \rightarrow \infty$ (a.s.) and, hence,

$$
\frac{s_{n}(a)}{X(n)} \sim \xi_{n}(a) \quad \text { a.s. }
$$

The following theorem is a simple consequence of Theorem 4.1.

Theorem 5.1. (i) If $\beta(a)=0$ then $s_{n}(a) / X(n) \stackrel{\mathrm{P}}{\rightarrow} \infty$.

(ii) If $0<\beta(a)<1$ then $s_{n}(a) / X(n) \stackrel{\mathrm{D}}{\rightarrow} G(\beta(a))$.

(iii) If $\beta(a)=1$ then $s_{n}(a) / X(n) \stackrel{\mathrm{P}}{\rightarrow} 0$. 
It should be noted that Theorems 4.3 and 4.4 can be restated as assertions about $s_{n}(a) / X(n)$. For example, if (4.3) holds for some $b>0$ and if $0<a \leq b$, then $s_{n}(a) / X(n) \rightarrow 0$ a.s.

The near-record sum $s_{n}(a)$ can be generalized as follows. Let $m$ be a natural number and let $\boldsymbol{a}=\left(a_{1}, \ldots, a_{m}\right)$ be a vector with all components positive. Let

$$
S_{n, m}(\boldsymbol{a})=s_{n}\left(a_{1}\right)+\cdots+s_{n+m-1}\left(a_{m}\right)
$$

be the total of near-record observations associated with the record values

$$
X(n), \ldots, X(n+m-1),
$$

i.e. between $L(n)$ and $L(n+m)$. Let $\xi_{n, m}(\boldsymbol{a})=\sum_{j=1}^{m} \xi_{n+j-1}\left(a_{j}\right)$. For $a_{1}=\cdots=a_{m}=a>$ 0 , we have

$$
(X(n)-a) \xi_{n, m}(\boldsymbol{a}) \leq S_{n, m}(\boldsymbol{a}) \leq X(n+m) \xi_{n, m}(\boldsymbol{a}) .
$$

Therefore, if $X(n+m) / X(n) \stackrel{\mathrm{P}}{\rightarrow} 1$, the asymptotic behaviour of $S_{n, m}(\boldsymbol{a})$ can be obtained from $\xi_{n, m}(\boldsymbol{a})$.

We now consider some results about quotients of record values, which are of interest in their own right.

Theorem 5.2. (a) If $\bar{F}(x) \in \mathcal{R}_{-\infty}$ then, for each $m=1,2, \ldots, X(n+m) / X(n) \stackrel{\mathrm{P}}{\rightarrow} 1$.

(b) Suppose, for all $b>1$, that

$$
K(b)=\int_{\mathbb{R}} \frac{\bar{F}(b x)}{\bar{F}^{2}(x)} \mathrm{d} F(x)<\infty .
$$

Then, for each $m=1,2, \ldots$,

$$
X(n+m) / X(n) \rightarrow 1 \text { a.s. }
$$

Proof. Observe that the sequence of record values comprises a Markov chain with a transition kernel given by (Nevzorov (2000, p. 68))

$$
\mathrm{P}\{X(n+1)>y \mid X(n)=x\}=\bar{F}(y) / \bar{F}(x) \quad(y \geq x) .
$$

Letting $y=b x$, where $b>1$, and integrating with respect to $F_{n}(x)$ gives

$$
\mathrm{P}\left\{\frac{X(n+1)}{X(n)}>b\right\}=\left(\int_{-\infty}^{x^{\prime}}+\int_{x^{\prime}}^{\infty}\right) \frac{\bar{F}(b x)}{\bar{F}(x)} \mathrm{d} F_{n}(x) .
$$

For a given $\varepsilon>0$, we can choose an $x^{\prime}$ so large that the integrand in the second integral is smaller than $\varepsilon$ and, hence, the right-hand side is bounded above by $F_{n}\left(x^{\prime}\right)+\varepsilon$. Letting $n \rightarrow \infty$ yields assertion (a) for $m=1$. If $m \geq 2$ then

$$
\frac{X(n+m)}{X(n)}=\frac{X(n+1)}{X(n)} \frac{X(n+2)}{X(n+1)} \cdots \frac{X(n+m)}{X(n+m-1)} \stackrel{\mathrm{p}}{\rightarrow} 1 .
$$

Next, summing over $n$ gives

$$
\sum_{n=1}^{\infty} \mathrm{P}\left\{\frac{X(n+m)}{X(n)}>b\right\}=K(b)
$$

(5.3) follows for $m=1$ from the Borel-Cantelli lemma, and the other cases follow as above. 
We now ask: under which conditions are the hypotheses of Theorem 5.2 satisfied? The rapid variation condition covers tail sizes ranging from thick tails of Weibull type through to medium and thin tails. That this condition is fundamental is suggested by the fact that if the integrand in (5.2) is nondecreasing, then $\bar{F}(b x) / \bar{F}(x) \rightarrow 0$ if $K(b)<\infty$. So, if (5.2) holds for all $b>1$ then $\bar{F}(x) \in \mathcal{R}_{-\infty}$. Another matter arises from the fact that the proof of part (b) shows (5.2) to be equivalent to complete convergence in (5.3), in the case of $m=1$. The $m$-step transition kernel of $\{X(n)\}$ can be used, as above, to show that sure convergence holds in (5.3) if and only if

$$
K_{m}(b)=\int_{\mathbb{R}} \frac{\bar{F}(b x)}{\bar{F}^{2}(x)}\left[-\log \frac{\bar{F}(b x)}{\bar{F}(x)}\right]^{m-1} \mathrm{~d} F(x)<\infty \quad(b>1) .
$$

Are these conditions equivalent? Given some technical conditions, the answer is 'yes'! The following result provides some answers to our questions by adding further structure to the rapid variation hypothesis. Our specific assumption for $\bar{F}(x) \in \mathcal{R}_{-\infty}$ is that

$$
\bar{F}(x)=(C(x) \exp [\rho(x)])^{-1},
$$

where $C(x) \rightarrow C>0, \rho(x)=\int_{1}^{x}(\delta(y) / y) \mathrm{d} y$ and the index function $\delta(y)$ is such that $\delta(y) \rightarrow$ $\infty$.

Theorem 5.3. (a) Suppose that $\beta(a)$ exists for all $a$. If $0 \leq \beta(a)<1$ then $\bar{F}(x) \in \mathcal{R}_{-\infty}$. If $\bar{F}(x)=(C(x) \exp [\rho(x)])^{-1}$, where $\rho(x) \in \overline{\mathcal{R}}_{\ell}$, and $0<\ell<1$ and $C(x) \rightarrow C>0$, then $\beta(a) \equiv 1$ and $\bar{F}(x) \in \mathcal{R}_{-\infty}$.

Suppose that $\bar{F}(x) \in \overline{\mathcal{R}}_{-\infty}$, where the index function $\delta(x)$ is eventually nondecreasing.

(b) If $\int_{1}^{\infty}(\delta(x))^{-\gamma} \mathrm{d} x<\infty$ for some $\gamma>0$, then (5.2) holds for all $b>1$.

(c) If, in addition, $\int_{x}^{b x}(\delta(y) / y) \mathrm{d} y=O(\delta(x))$ for all $b>1$, then $K_{m}(b)<\infty$ holds for all $b, m>1$.

Proof. If $b>1$ and $a>0$, then $b x>x+a$ if $x \geq a /(b-1)$, and (4.1) implies that $\lim \sup _{x \rightarrow \infty} \bar{F}(b x) / \bar{F}(x) \leq \beta(a)$. However, if $\beta(a)<1$ then $\beta(a) \rightarrow 0$ as $a \rightarrow \infty$, implying that $\bar{F}(x) \in \mathcal{R}_{-\infty}$. The second part of assertion (a) follows by straightforward calculation.

The normalization assumption implies that $F^{\prime}(x)=(\delta(x) / x) \bar{F}(x)$ and, since

$$
\rho(b x)-\rho(x) \geq \delta(x) \log b
$$

for sufficiently large $x$, we find that (5.2) holds if $\int_{1}^{\infty} \delta(x) \mathrm{e}^{-A \delta(x)} \mathrm{d} x<\infty$. However, the exponential term is dominated by $k !(A \delta(x))^{-k}$ for any integer $k \geq \gamma+1$, and assertion (b) follows.

The additional hypothesis of assertion (c) implies that $\rho(b x)-\rho(x)=O(\delta(x))$, which, together with estimates in the proof of assertion (b), implies that $K_{m}(b)<\infty$ if

$$
\int_{1}^{\infty}(\delta(x))^{m} \mathrm{e}^{-A \delta(x)} \mathrm{d} x<\infty .
$$

Assertion (c) now follows similarly to assertion (b), if we choose $k>\gamma+m$.

Assertions (b) and (c) hold if there exist positive constants $c, d$, and $x^{\prime}$, with $c<d$, such that $x^{c}<\delta(x)<x^{d}$ if $x \geq x^{\prime}$ or if $\delta(x)$ has faster than polynomial growth. The assumptions of assertion (b) are sharp in that examples will show that (5.2) may or may not hold if $\delta(x) \in \mathcal{R}_{0}$.

Our next result shows that record value gaps increase unboundedly under a suitable condition. 
Theorem 5.4. (a) If $\bar{F}(x) \in \mathcal{R}_{0}$ then, for $m=1,2, \ldots, X(n+m) / X(n) \stackrel{\mathrm{P}}{\rightarrow} \infty$.

(b) Suppose, for all $b>1$, that

$$
\bar{I}(b)=\int_{\mathbb{R}} \frac{\bar{F}(x)-\bar{F}(b x)}{\bar{F}^{2}(x)} \mathrm{d} F(x)<\infty .
$$

Then, for each $m=1,2, \ldots, X(n+m) / X(n) \rightarrow \infty$ a.s.

Proof. The assertions for $m=1$ follow as in the proof of Theorem 5.2, upon observing that

$$
\mathrm{P}\{X(n+1) \leq b X(n)\}=\int_{\mathbb{R}} \frac{1-\bar{F}(b x)}{\bar{F}(x)} \mathrm{d} F_{n}(x) \rightarrow 0
$$

and

$$
\sum_{n=1}^{\infty} \mathrm{P}\{X(n+1) \leq b X(n)\}=\bar{I}(b),
$$

and invoking the Borel-Cantelli lemma. For $m \geq 2$, observe that

$$
\{X(n+m) \leq b X(n+1)\} \subset\{X(n+1) \leq b X(n+1)\},
$$

from which the remainder of the proof follows.

If $b>1$ then $x+a<b x$ for any $a$ and large enough $x$. It follows that if (5.4) holds for fixed $b>1$, then $I(a)<\infty$ (see (4.3)) holds for all $a>0$; whence $\beta(a) \equiv 1$. Thus, (5.4) determines a subset of thick-tailed distributions. In fact, if (5.4) holds for all $b>1$ and the integrand is eventually nondecreasing, then $(\bar{F}(x)-\bar{F}(b x)) / \bar{F}(x) \rightarrow 0$, i.e. $\bar{F}(x) \in \mathcal{R}_{0}$. As a partial converse, suppose that $\bar{F}(x) \in \overline{\mathcal{R}}_{0}$ with index function $-\epsilon(x)$ (which must satisfy $\left.\int_{1}^{\infty}(\epsilon(x) / x) \mathrm{d} x=\infty\right)$ : then $F(x)$ has density function $\epsilon(x) \bar{F}(x) / x$. If we further assume that $\epsilon(x)$ is eventually nonincreasing, or that $\epsilon(x) \in \mathcal{R}_{0}$, then $1-\bar{F}(b x) / \bar{F}(x)=O(\epsilon(x))$ and it follows that (5.4) holds if $\int_{1}^{\infty}\left(\epsilon^{2}(x) / x\right) \mathrm{d} x<\infty$.

Our next result extends Theorem 4.1 and will be used to obtain the asymptotic behaviour of $S_{n, m}(\boldsymbol{a})$.

Theorem 5.5. Suppose that $a_{i}>0$ and $\beta\left(a_{i}\right)$ exist for $i=1, \ldots, m$, where $m$ is a natural number. Then

$$
\left(\xi_{n}\left(a_{1}\right), \ldots, \xi_{n+m-1}\left(a_{m}\right)\right) \stackrel{\mathrm{D}}{\rightarrow}\left(G\left(\beta\left(a_{1}\right)\right), \ldots, G\left(\beta\left(a_{m}\right)\right)\right),
$$

where $G(\gamma)$ is a geometrically distributed random variable with parameter $\gamma$ and the components of the limit random vector are independent. In particular, $\xi_{n, m}(\boldsymbol{a}) \stackrel{\mathrm{D}}{\rightarrow} G_{m}(\boldsymbol{a})$, where

$$
\mathrm{E}\left[t^{G_{m}(\boldsymbol{a})}\right]=\prod_{i=1}^{m} \frac{\beta\left(a_{i}\right)}{1-\left(1-\beta\left(a_{i}\right)\right) t} \quad(0 \leq t<1) .
$$

Proof. Theorem 2.2 implies that, given $X(n), \ldots, X(n+m-1)$, the counts $\xi_{n+i-1}\left(a_{i}\right)$, $i=1, \ldots, m$, are conditionally independent with a geometric distribution, and that their joint probability-generating function is

$$
\mathrm{E}\left[\prod_{i=1}^{m} t_{i}^{\xi_{n+i-1}\left(a_{i}\right)}\right]=\mathrm{E}\left[\prod_{i=1}^{m} \frac{\beta\left(a_{i}, X(n+i-1)\right)}{1-\left(1-\beta\left(a_{i}, X(n+i-1)\right)\right) t_{i}}\right] \quad\left(0 \leq t_{i}<1\right) .
$$

The assertion follows from the dominated convergence theorem, since $\beta\left(a_{i}, X(n+i-1)\right) \rightarrow$ $\beta\left(a_{i}\right)$ a.s. 
The distribution of the weak limit $G_{m}(\boldsymbol{a})$ is a convolution of geometric laws that can be expressed as a weighted sum of geometric and negative binomial distributions via the partial fraction expansion of the right-hand side of (5.5). In particular, if the components of $\boldsymbol{a}$ are all different then

$$
\mathrm{P}\left\{G_{m}(\boldsymbol{a})=k\right\}=\sum_{i=1}^{m} \alpha_{i}(m) \beta\left(a_{i}\right)\left(1-\beta\left(a_{i}\right)\right)^{k} \quad(k=0,1, \ldots),
$$

where

$$
\alpha_{i}(m)=\left(1-\beta\left(a_{i}\right)\right)^{m-1} \prod_{j=1, j \neq i} \frac{\beta\left(a_{j}\right)}{\beta\left(a_{j}\right)-\beta\left(a_{i}\right)} .
$$

This is a trivial consequence of (5.5), but see Sen and Balakrishnan (1999) for a different approach. Note that $\sum_{i=1}^{m} \alpha_{i}(m)=1$, but that both signs occur among the $\alpha_{i}(m)$. If $\ell$ of the $a_{i}$ have the same value $a_{\ell}, \ell \geq 2$, then (5.6) must be augmented with negative binomial probabilities $\left(\begin{array}{c}v-1+k \\ k\end{array}\right)\left(\beta\left(a_{\ell}\right)\right)^{\nu}\left(1-\beta\left(a_{\ell}\right)\right)^{k}$ for $v=1, \ldots, \ell$. This is also a direct consequence of the partial fraction expansion of (5.5).

Combining Theorems 5.2(a) and 5.5 with (5.1) yields the following result.

Theorem 5.6. Suppose that $\bar{F}(x) \in \mathcal{R}_{-\infty}$ and that $a_{i}>0$ and $\beta\left(a_{i}\right)$ exist for $i=1, \ldots, m$, where $m$ is a natural number. Then $S_{n, m}(\boldsymbol{a}) / X(n) \stackrel{\mathrm{D}}{\rightarrow} G_{m}(\boldsymbol{a})$, which has probability-generating function (5.5).

(a) In particular, if $0<\beta\left(a_{i}\right)<1$ and

(i) if the components of a all differ, then the limit distribution is given explicitly by (5.6) and (5.7);

(ii) if $a_{1}=\cdots=a_{m}$, then

$$
\mathrm{P}\left\{G_{m}=k\right\}=\left(\begin{array}{c}
m-1+k \\
k
\end{array}\right)(\beta(a))^{m}(1-\beta(a))^{k} \quad(k=0,1, \ldots) .
$$

(b) If $\beta(a) \equiv 0$ with $a>0$ then, for any vector a with positive components, we have

$$
\mathrm{P}\left\{G_{m}(\boldsymbol{a})=\infty\right\}=1 .
$$

(c) If $\beta(a) \equiv 1$ with $a>0$ then, for any vector $\boldsymbol{a}$ with positive components, we have

$$
\mathrm{P}\left\{G_{m}(\boldsymbol{a})=0\right\}=1 .
$$

\section{Examples}

Example 6.1. The standard Cauchy distribution has density function $f(x)=\left[\pi\left(1+x^{2}\right)\right]^{-1}$ and, hence, $\bar{F}(x) \in \mathcal{R}_{-1}$, a thick tail. Consequently, Theorems 4.1(iii), 4.2(iii), and 5.1(iii) hold. In addition,

$$
\bar{F}(x-b)-\bar{F}(x)=\pi^{-1} \int_{x-b}^{x} \frac{\mathrm{d} y}{1+y^{2}} \sim \frac{b}{\pi x^{2}}=O\left(\bar{F}^{2}(x)\right)
$$

and, hence, the integrand in (4.3) is $O(1)$. Thus, the assertions of Theorem 4.3 hold for Cauchy distributions. On the other hand, Theorem 5.4 does not apply for Cauchy tails. 
Example 6.2. Similar considerations show that any distribution with a Pareto tail, $\bar{F}(x) \sim$ const. $x^{-\alpha}$ with $\alpha>0$, satisfies the conditions of Theorems 4.1(iii), 4.2(iii), and 5.1(iii), but not those of Theorem 5.4. Li (1999) showed that (4.3) holds for any $b>0$ in the Pareto tail case, and Pakes showed this for $\bar{F}(x) \in \mathcal{R}_{-\alpha}$.

Example 6.3. Let $F(x)=1-\log 2 / \log x \in \overline{\mathcal{R}}_{0}, x>\log 2$. It follows by direct calculation that Theorem 5.4 holds. Alternatively, observe that the index function $\epsilon(x) \propto 1 / \log x$ satisfies the conditions following the proof of Theorem 5.4. Thus, we have the following almost-sure convergences:

$$
\xi_{n}(a) \rightarrow 0, \quad s_{n}(a) / X(n) \rightarrow 0, \quad X(n+1) / X(n) \rightarrow \infty .
$$

Example 6.4. The Weibull-type survivor function $F_{\alpha}(x)=1-\mathrm{e}^{-x^{\alpha}}, x>0, \alpha>0$, covers the range of thin to thick tails. The index function is $\delta(x)=\alpha x^{\alpha}$, so $\bar{F}(x) \in \overline{\mathcal{R}}_{-\infty}$ and it is obvious that the conditions of parts (b) and (c) of Theorem 5.3 hold. Hence, Theorem 5.3 holds with sure convergence for all $\alpha>0$ and $m$. Next, since

$$
a \alpha(x-a)^{\alpha-1}<x^{\alpha}-(x-a)^{\alpha}<a \alpha x^{\alpha-1},
$$

the following classification is evident.

(I) If $\alpha>1$ then $F$ is thin tailed and, since $F^{\prime}(x)=\alpha x^{\alpha-1} \bar{F}(x)$, it is clear that

$$
J(b)=O\left(\int_{1}^{\infty} x^{\alpha-1}\left[\exp \left(-b \alpha(x-b)^{\alpha-1}\right)\right] \mathrm{d} x\right)<\infty
$$

in (4.4). Hence, Theorems 4.1(i), 4.2(i), 4.4, 5.1(i), and 5.6(c) hold.

(II) If $\alpha=1$ then $F(x)$ is the standard exponential distribution function, which is medium tailed. Hence, Theorems 4.1(ii), 4.2(ii), 5.1(ii), and 5.6(a) hold.

(III) If $0<\alpha<1$ then $F(x)$ is thick tailed and Theorem 5.6(c) holds. In addition, (4.3) is satisfied for all $b$ if $0<\alpha<\frac{1}{2}$, and is never satisfied if $\frac{1}{2} \leq \alpha<1$.

Example 6.5. The standard normal distribution function $\Phi(x)$ satisfies $\bar{\Phi}(x) \sim \phi(x) / x$, $x \rightarrow \infty$, where $\phi(x)=\Phi^{\prime}(x)$. Referring to Theorem 5.3, we see that $\delta(x)=\frac{1}{2} x^{2(1+o(1))}$ and clearly $\beta(a) \equiv 1$. Consequently, the conditions of Theorem 5.3(c) are satisfied. In addition, Theorems 5.1(i) and 5.6(c) hold. Finally, $-\log \Phi(x)=\frac{1}{2} x^{2}+\log x+O(1)$, whence the conditions of Theorem 4.5(b) are satisfied with $\ell=2$. It follows that Theorems 4.1(i), 4.2(i), and 4.4 hold.

Example 6.6. Here we prove the claim made in the remarks following Theorem 4.4. Suppose that $\bar{F}(x)=\exp [-x L(x)]$, where $L(x) \in \overline{\mathcal{R}}_{0}$ and $L(x) \rightarrow \infty$ as $x \rightarrow \infty$. If $a$ is real then

$$
\begin{aligned}
x L(x)-(x-a) L(x-a) & =a L(x)-(x-a)(L(x)-L(x-a)) \\
& =a L(x)(1+o(1)) \\
& \rightarrow \infty
\end{aligned}
$$

as $x \rightarrow \infty$. Hence, $\beta(a) \equiv 0$ and Theorems 4.1(i), 4.2(i), and 5.1(i) hold. Since

$$
F^{\prime}(x)=\bar{F}(x) L(x)(1+o(1)),
$$


we infer that (4.4) holds if

$$
\hat{J}(b)=\int_{\mathrm{e}}^{\infty} L(x) \mathrm{e}^{-b L(x)} \mathrm{d} x<\infty .
$$

If we further assume that $x L^{\prime}(x)=O(1)$, then (4.4) holds if and only if (6.1) does.

For the specific case $L(x)=(\log x)^{c}, c>0, x \geq \mathrm{e}$, some calculation yields

$$
\hat{J}(b)=c^{-1} \int_{1}^{\infty} y^{1 / c} \exp \left[-b y+y^{1 / c}\right] \mathrm{d} y \begin{cases}=\infty & \text { for all } b>0 \text { if } c<1, \\ <\infty & \text { if } c=1 \text { and then if and only if } b>1, \\ <\infty & \text { for all } b>0 \text { if } c>1 .\end{cases}
$$

Example 6.7. Referring to the remarks following the proof of Theorem 5.3, suppose that $\bar{F}(x) \in \mathcal{R}_{-\infty}$ with index function $\delta(x)=1+\log (\log x)$ for $x \geq \mathrm{e}$. With $\ell:=\log b>0$, we find that

$$
\rho(b x)-\rho(x)=\ell+\int_{\log x}^{\ell+\log x} \log y \mathrm{~d} y=\ell(1+\log (\log x))+o(1) \rightarrow \infty
$$

and, hence, $\beta(b) \equiv 0$. Since $F^{\prime}(x)=(\delta(x) / x) \bar{F}(x)$, we have

$$
K(b)=b \int_{\mathrm{e}}^{\infty} x^{-1}(1+\log (\log x))(\log x)^{-\ell} \mathrm{d} x+O(1)=b \int_{1}^{\infty}(1+\log y) y^{-\ell} \mathrm{d} y+O(1) .
$$

Hence, $K(b)<\infty$ if and only if $b>$ e. On the other hand, the slowly varying index function $\delta(x)=\log x$ yields $K(b)<\infty$ for all $b>1$.

\section{Acknowledgement}

The first author thanks the Natural Sciences and Engineering Research Council of Canada for funding this research.

\section{References}

Arnold, B. C. and Villasenor, J. A. (1998). The asymptotic distributions of sums of records. Extremes 1, 351-363. Arnold, B. C., Balakrishnan, N. And Nagaraja, H. N. (1998). Records. John Wiley, New York.

Balakrishnan, N. And Stepanov, A. (2005). A note on the number of observations near an order statistic. J. Statist. Planning Infer. 134, 1-14.

Baryshnikov, Y., Eisenberg, B. and Stengle, G. (1995). A necessary and sufficient condition for the existence of the limiting probability of a tie for first place. Statist. Prob. Lett. 23, 203-209.

Bingham, N. H., Goldie, C. and Teugels, J. F. (1987). Regular Variation. Cambridge University Press.

Brands, J. J. A. M., Steutel, F. W. And Wilms, R. J. G. (1994). On the number of maxima in a discrete sample. Statist. Prob. Lett. 20, 209-217.

Eisenberg, B., Stengle, G. and Strang, G. (1993). The asymptotic probability of a tie for first place. Ann. Appl. Prob. 3, 731-745.

Galambos, J. (1987). The Asymptotic Theory of Extreme Order Statistics, 2nd edn. Krieger, Melbourne, FL.

HASHORVA, E. (2003). On the number of near-maximum insurance claims under dependence. Insurance Math. Econom. 32, 37-43.

Hu, Z. AND Su, C. (2003). Limit theorems for the number and sum of near-maxima for medium tails. Statist. Prob. Lett. 63, 229-237.

LI, Y. (1999). A note on the number of records near the maximum. Statist. Prob. Lett. 43, 153-158.

LI, Y. AND PAKes, A. G. (2001). On the number of near-maximum insurance claims. Insurance Math. Econom. 28, 309-318.

Nevzorov, V. B. (1986). On $k$ th record moments and generalizations. Zapiski Nauchn Sem. LOMI 153, $115-121$ (in Russian). 
Nevzorov, V. B. (2000). Records: Mathematical Theory (Transl. Math. Monogr. 194). American Mathematical Society, Providence, RI.

Pakes, A. G. (2000). The number and sum of near-maxima for thin-tailed populations. Adv. Appl. Prob. 32, $1100-1116$. PAKES, A. G. (2005). Criteria for convergence of the number of near maxima for long tails. To appear in Extremes.

PAKes, A. G. AND LI, Y. (1998). Limit laws for the number of near maxima via the Poisson approximation. Statist. Prob. Lett. 40, 395-401.

Pakes, A. G. And Steutel, F. W. (1997). On the number of records near the maximum. Austral. J. Statist. 39, $179-193$. QI, Y. (1997). A note on the number of maxima in a discrete sample. Statist. Prob. Lett. 33, 373-377.

Sen, A. and Balakrishnan, N. (1999). Convolution of geometrics and a reliability problem. Statist. Prob. Lett. 43, 421-426.

Stepanov, A. V. (1992). Limit theorems for weak records. Theory Prob. Appl. 37, 570-574.

Stepanov, A. V., Balakrishnan, N. and Hofmann, G. (2003). Exact distribution and Fisher information of weak record values. Statist. Prob. Lett. 64, 69-81. 